\title{
Random matrix analysis of the QCD sign problem*
}

\section{Jacques Bloch and Tilo Wettig}

Institute for Theoretical Physics, University of Regensburg, 93040 Regensburg, Germany

E-mail: jacques.bloch@physik.uni-regensburg.de,

tilo.wettig@physik.uni-regensburg.de

The severity of the sign problem in lattice QCD at nonzero baryon density is measured by the average phase of the fermion determinant. Motivated by the equivalence of chiral random matrix theory and QCD to leading order in the epsilon regime, we compute the phase of the fermion determinant for general topology in random matrix theory as a function of the quark chemical potential and the quark mass. We find that the sign problem becomes milder with increasing topological charge. The analytic predictions are verified by detailed numerical random matrix simulations.

The XXVII International Symposium on Lattice Field Theory

July 26-31, 2009

Peking University, Beijing, China

*Supported by the German Research Foundation (DFG).

†peaker. 


\section{Introduction}

The fermion sign problem is a major obstacle for unquenched lattice QCD simulations at nonzero density, see [1] for a review at this conference. The severity of the sign problem is measured by the average phase of the fermion determinant. Let us denote the Dirac operator by $D(m ; \mu)$, where $m$ and $\mu$ are the quark mass and the quark chemical potential, respectively, and write det $D(m ; \mu)=r e^{i \theta} \in \mathbb{C}$. Then the average phase of the two-flavor theory is given by $\left\langle e^{2 i \theta}\right\rangle$. It was shown in [2] that the average phase is nonzero for $\mu<m_{\pi} / 2$, whereas for $\mu>m_{\pi} / 2$ it is exponentially suppressed in $\mu^{2} F^{2} V$. Here, $m_{\pi}$ is the pion mass, $V$ is the volume, and $F$ and $\Sigma$ (see (1.1) below) are the familiar low-energy constants in the effective chiral Lagrangian.

The microscopic regime of QCD (corresponding to the leading order in the $\varepsilon$-expansion) is defined by the requirement that the scaling variables

$$
\hat{m}=m V \Sigma \quad \text { and } \quad \hat{\mu}^{2}=\mu^{2} F^{2} V
$$

are kept fixed in the $V \rightarrow \infty$ limit. In this regime exact analytical results for QCD can be derived using chiral random matrix theory (RMT). In [3] the average phase was computed for zero topology from RMT. An interesting question (e.g., for fixed-topology simulations [4], which could also be done at $\mu \neq 0$ using the overlap operator [5]) is whether the sign problem becomes harder or milder as a function of the topological charge $v$. In [6] we have generalized the results of [3] to nonzero $v$. In this contribution, we summarize our main results and present some additional material.

Before doing so, let us briefly mention important work related to the present subject. The leading-order $p$-regime corrections to the RMT result for the average phase were computed in [3]. The average phase at nonzero temperature (but still at $v=0$ ) was computed from RMT in [7]. The distribution of the phase was studied in [8] (see also [9] at this conference), and lattice studies of the phase were performed in $[10,11]$.

\section{Random matrix model and sketch of the calculation}

The starting point of our calculation is the non-hermitian random matrix model [12]

$$
D(m ; \mu)=\left(\begin{array}{cc}
m & i \Phi_{1}+\mu \Phi_{2} \\
i \Phi_{1}^{\dagger}+\mu \Phi_{2}^{\dagger} & m
\end{array}\right)
$$

for the Dirac operator, where $\Phi_{1}$ and $\Phi_{2}$ are independent complex random matrices of dimension $(N+v) \times N$, distributed according to the same Gaussian distribution, $P(X) \propto \exp \left(-N \operatorname{tr} X X^{\dagger}\right)$. The matrix in (2.1) has $|v|$ exact zero modes, which allows us to identify $v$ with the topological charge. We keep $v$ fixed and take $N \rightarrow \infty$. Without loss of generality we assume $v \geq 0$; for $v<0$ we can simply replace $v$ by $|v|$ in our results.

Going over to an eigenvalue representation of the random matrices $\Phi_{1}$ and $\Phi_{2}$, the partition function of the random matrix model with $N_{f}$ flavors becomes [12]

$$
Z_{v}^{N_{f}}(\alpha)=\int_{\mathbb{C}} \prod_{k=1}^{N} d^{2} z_{k} w^{v}\left(z_{k}, z_{k}^{*} ; \alpha\right)\left|\Delta_{N}\left(\left\{z^{2}\right\}\right)\right|^{2} \prod_{f=1}^{N_{f}}\left(m_{f}^{2}-z_{k}^{2}\right),
$$


where $\alpha=\mu^{2}, \Delta_{N}$ is the Vandermonde determinant, and we have introduced the weight function

$$
w^{v}\left(z, z^{*} ; \alpha\right)=|z|^{2 v+2} \exp \left(-\frac{N(1-\alpha)}{4 \alpha}\left(z^{2}+z^{* 2}\right)\right) K_{v}\left(\frac{N(1+\alpha)}{2 \alpha}|z|^{2}\right)
$$

that includes a modified Bessel function $K$. The ensemble average of an observable is given in the usual way by including it in the integrand and dividing the resulting integral by $Z$.

The phase factor of the squared determinant can be written as

$$
e^{2 i \theta}=\frac{\operatorname{det}(D(\mu)+m)}{\operatorname{det}\left(D^{\dagger}(\mu)+m\right)}=\prod_{k=1}^{N} \frac{m^{2}-z_{k}^{2}}{m^{2}-z_{k}^{* 2}}
$$

with ensemble average

$$
\begin{aligned}
\left\langle e^{2 i \theta}\right\rangle_{N_{f}} & =\left\langle\frac{\operatorname{det}(D(\mu)+m)}{\operatorname{det}\left(D^{\dagger}(\mu)+m\right)}\right\rangle_{N_{f}}=\frac{Z_{v}^{N_{f}+1 \mid 1^{*}}(\alpha, m)}{Z_{v}^{N_{f}}(\alpha)} \\
& =\frac{1}{Z_{v}^{N_{f}}(\alpha)} \int_{\mathbb{C}} \prod_{k=1}^{N} d^{2} z_{k} w^{v}\left(z_{k}, z_{k}^{*} ; \alpha\right)\left|\Delta_{N}\left(\left\{z^{2}\right\}\right)\right|^{2} \frac{m^{2}-z_{k}^{2}}{m^{2}-z_{k}^{* 2}} \prod_{f=1}^{N_{f}}\left(m_{f}^{2}-z_{k}^{2}\right),
\end{aligned}
$$

where $Z_{v}^{N_{f}+1 \mid 1^{*}}$ is the RMT partition function with $N_{f}+1$ fermionic quarks and one conjugate bosonic quark. The integrals in (2.2) and (2.5) can be done for any $N$ using the orthogonalpolynomial formalism developed in $[13,14]$. The resulting calculation is too extensive to be reproduced here, and we therefore refer to [6] for the details. The main ingredients are orthogonal polynomials [12] with respect to the weight function (2.3) and their Cauchy transforms. After taking the microscopic limit with the RMT scaling variables $\hat{m}=2 \mathrm{Nm}$ and $\hat{\alpha}=2 \mathrm{N \alpha}$ (corresponding to the physical scaling variables in (1.1)) kept fixed, one arrives at

$$
\left\langle e_{s}^{2 i \theta}\right\rangle_{N_{f}}=\frac{1}{(2 \hat{m})^{N_{f} N_{f} !}} \frac{\left|\begin{array}{cccc}
\mathscr{H}_{v, 0}^{s}(\hat{\alpha}, \hat{m}) & \mathscr{H}_{v, 1}^{s}(\hat{\alpha}, \hat{m}) & \cdots & \mathscr{H}_{v, N_{f}+1}^{s}(\hat{\alpha}, \hat{m}) \\
I_{v, 0}(\hat{m}) & I_{v, 1}(\hat{m}) & \cdots & I_{v, N_{f}+1}(\hat{m}) \\
I_{v, 0}^{\prime}(\hat{m}) & I_{v, 1}^{\prime}(\hat{m}) & \cdots & I_{v, N_{f}+1}^{\prime}(\hat{m}) \\
\vdots & \vdots & \vdots & \vdots \\
I_{v, 0}^{\left(N_{f}\right)}(\hat{m}) & I_{v, 1}^{\left(N_{f}\right)}(\hat{m}) & \cdots & I_{v, N_{f}+1}^{\left(N_{f}\right)}(\hat{m})
\end{array}\right|}{\left|\begin{array}{cccc}
I_{v, 0}(\hat{m}) & I_{v, 1}(\hat{m}) & \cdots & I_{v, N_{f}-1}(\hat{m}) \\
I_{v, 0}^{\prime}(\hat{m}) & I_{v, 1}^{\prime}(\hat{m}) & \cdots & I_{v, N_{f}-1}^{\prime}(\hat{m}) \\
\vdots & \vdots & \vdots & \vdots \\
I_{v, 0}^{\left(N_{f}-1\right)}(\hat{m}) & I_{v, 1}^{\left(N_{f}-1\right)}(\hat{m}) & \cdots & I_{v, N_{f}-1}^{\left(N_{f}-1\right)}(\hat{m})
\end{array}\right|}
$$

where the subscript $s$ labels the microscopic limit, we have assumed degenerate quark masses, $I_{v, k}(z)=z^{k} I_{v+k}(z)$ with modified Bessel function $I$, and

$$
\mathscr{H}_{v, k}^{s}(\hat{\alpha}, \hat{m})=-\frac{e^{-2 \hat{\alpha}}}{4 \pi \hat{\alpha} \hat{m}^{v}} \int_{\mathbb{C}} \frac{d^{2} z}{z^{2}-\hat{m}^{2}} \frac{|z|^{2(v+1)}}{z^{* V}} \exp \left(-\frac{z^{2}+z^{* 2}}{8 \hat{\alpha}}\right) K_{V}\left(\frac{|z|^{2}}{4 \hat{\alpha}}\right) I_{V, k}\left(z^{*}\right)
$$


corresponds to the microscopic limit of the Cauchy transform mentioned above. The calculation of this integral is a nontrivial exercise in complex analysis and results in

$$
\begin{aligned}
\mathscr{H}_{v, k}^{s}(\hat{\alpha}, \hat{m})=\frac{e^{-2 \hat{\alpha}-\frac{\hat{m}^{2}}{8 \tilde{\alpha}}}}{4 \hat{\alpha}}\left[\int_{0}^{\infty} d u(-)^{k} u^{k+1} \exp \left(-\frac{u^{2}}{8 \hat{\alpha}}\right) I_{v}\left(\frac{\hat{m} u}{4 \hat{\alpha}}\right) K_{v+k}(u)\right. \\
\left.+\int_{0}^{\hat{m}} d u u^{k+1} \exp \left(-\frac{u^{2}}{8 \hat{\alpha}}\right) K_{v}\left(\frac{\hat{m} u}{4 \hat{\alpha}}\right) I_{v+k}(u)\right]+\Delta_{v, k}(\hat{\alpha}, \hat{m})
\end{aligned}
$$

with

$$
\Delta_{v, k}(\hat{\alpha}, \hat{m})=e^{-2 \hat{\alpha}-\frac{\hat{m}^{2}}{8 \hat{\alpha}}} \frac{(-)^{k} 2^{v+k-1}}{\hat{m}^{v}} \sum_{i, j=0}^{i+j \leq v-1} \frac{(v-1-i) !(v+k-1-j) !}{(v-1-i-j) ! i ! j !}(2 \hat{\alpha})^{j}\left(\frac{\hat{m}^{2}}{8 \hat{\alpha}}\right)^{i}
$$

The $v=0$ limit of (2.8) agrees with the result obtained in [3], and $\Delta_{v, k}$ is a new term that was not present for $v=0$. This essentially completes the calculation.

From the above equations one can derive a number of interesting limits, such as the chiral or the thermodynamic limit. In the latter limit, we take $\hat{m}$ and $\hat{\alpha}$ to infinity keeping their ratio fixed and obtain

$$
\left\langle e_{s}^{2 i \theta}\right\rangle^{\text {th }}= \begin{cases}(1-2 \hat{\alpha} / \hat{m})^{N_{f}+1} & \text { for } 2 \hat{\alpha}<\hat{m} \\ 0 & \text { for } 2 \hat{\alpha}>\hat{m}\end{cases}
$$

where the condition $2 \hat{\alpha}<\hat{m}$ corresponds to $\mu<m_{\pi} / 2$. Note that (2.10) is independent of $v$ and agrees with the result of $[2,3]$.

\section{Results and discussion}

In Fig. 1 we display the dependence of the average phase in the microscopic limit on the parameters of the problem. As expected, the sign problem becomes harder as we increase $\hat{\alpha}$ (i.e., $\mu$ ) and decrease $\hat{m}$ (i.e., $m$ ).

The dependence of $\left\langle e_{s}^{2 i \theta}\right\rangle$ on $v$ can be anticipated based on a qualitative argument. At $\mu=0$ the Dirac eigenvalues are purely imaginary, and at $\mu \neq 0$ they move away from the imaginary axis into the complex plane. The behavior of the small nonzero Dirac eigenvalues is known from RMT $[12,15]$. On average, the imaginary part of the small eigenvalues increases with $|v|$, whereas the real part is mainly controlled by $\mu$ and depends only weakly on $v$. Therefore, we expect the average phase to increase with $|v|$. This expectation is confirmed by the analytical results displayed in Fig. 1, i.e., the sign problem becomes milder as $|v|$ is increased (as expected, this effect weakens if $\hat{m}$ is increased). This may be an interesting observation for groups doing lattice simulations at fixed topology in the $\varepsilon$-regime of QCD [16]. As long as the parameter $\hat{\mu}$ in (1.1) is not too large, unquenched simulations using reweighting techniques (see Sec. 4 below) are feasible. It would then be advantageous to simulate not at $v=0$ but at larger values of $v$.

As for the dependence on $N_{f}$, one would naively expect that more flavors make the sign problem harder. However, there is a competing effect, again based on the average behavior of the small eigenvalues known from RMT: For small $\hat{m}, N_{f}$ has the same effect on them as $|v|$, i.e., it increases 

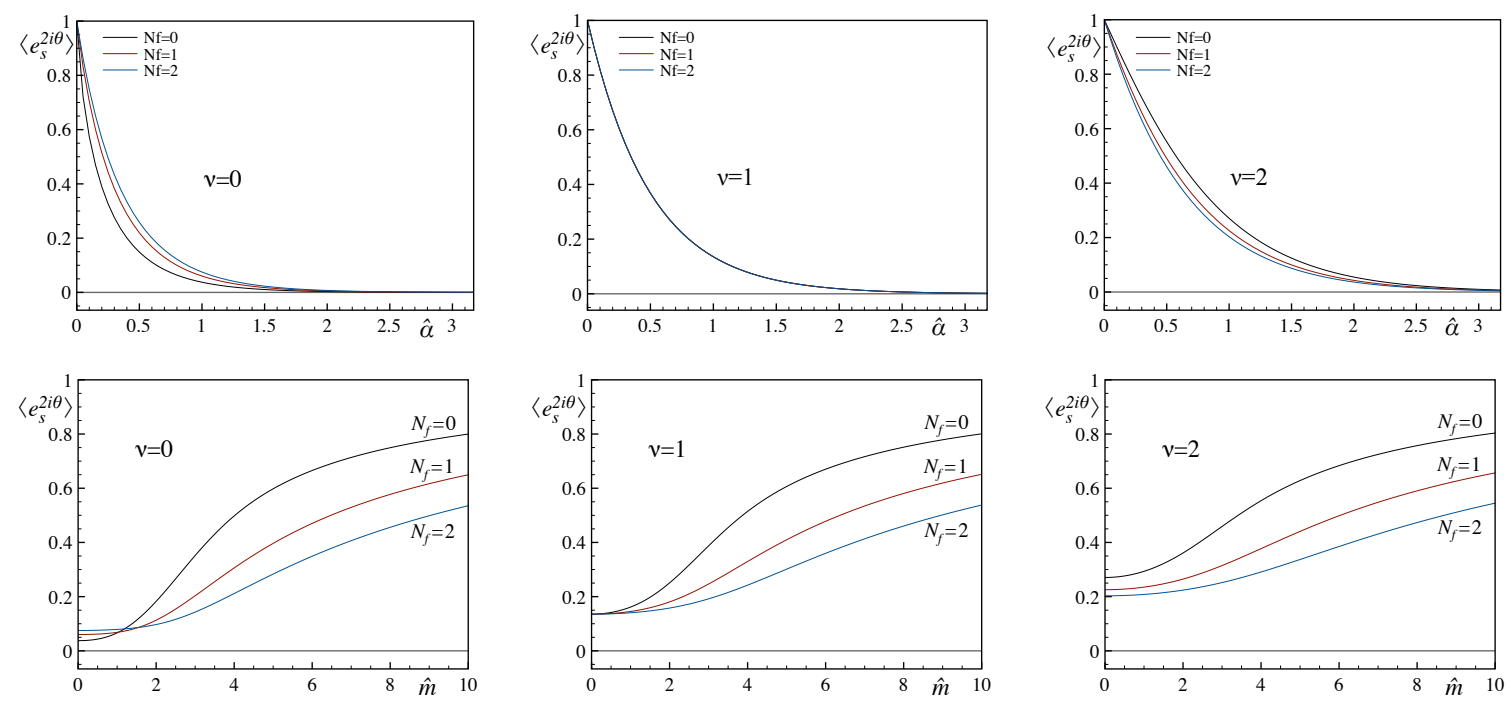

Figure 1: The top row shows the $\hat{\alpha}$-dependence of the average phase in the chiral limit for $v=0,1,2$ and $N_{f}=0,1,2$. The bottom row shows the dependence on $\hat{m}$ for fixed $\hat{\alpha}=1$ and the same values of $v$ and $N_{f}$.

the imaginary part but does not significantly affect the real part. As above, this competing effect is washed out if $\hat{m}$ is increased. The $N_{f}$-dependence of $\left\langle e_{s}^{2 i \theta}\right\rangle$ in Fig. 1 is consistent with this qualitative argument. We observe that for $v=0$ and small $\hat{m}$, increasing $N_{f}$ actually makes the sign problem milder. Interestingly, for $|v|=1$ and in the chiral limit, $\left\langle e_{s}^{2 i \theta}\right\rangle$ is completely independent of $N_{f}$. For $v>0$ the competing effect of $N_{f}$ does not dominate, i.e., increasing $N_{f}$ makes the sign problem harder as naively expected.

\section{Numerical random matrix simulations}

We have confirmed our analytical results (including finite- $N$ effects) by extensive numerical simulations of random matrices [6]. Examples are shown in Fig. 2 (for the quenched case) and in Fig. 3 (for $N_{f}=2$ ). In the unquenched case, the numerical data were obtained by three different reweighting methods:

1. reweighting from the quenched ensemble,

2. reweighting from the phase-quenched ensemble (with $r=|\operatorname{det} D(m ; \mu)|$ in the measure),

3. reweighting from the "sign-quenched" ensemble (with $r|\cos \theta|$ and $r|\sin \theta|$ in the measure).

Method 3 minimizes the amount of reweighting that is being done and is therefore expected to lead to the smallest statistical errors. This expectation is confirmed in the right plot in Fig. 3.

It would now be interesting to compare our analytical results with data from lattice simulations. We have done initial lattice studies, but our volume was too small to find agreement (more precisely, we did not have enough eigenvalues in the microscopic regime). In the future we hope to be able to go to larger volumes to confirm the validity of the RMT results for QCD. 

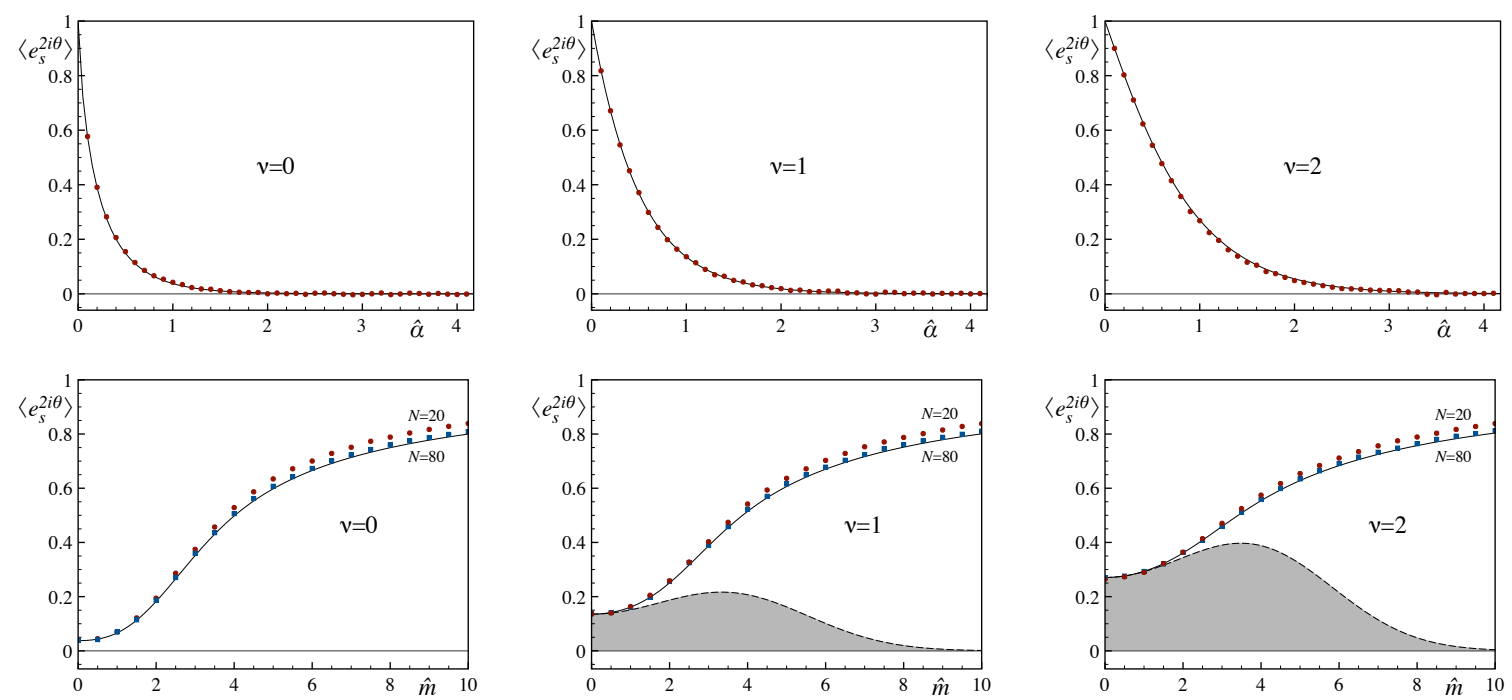

Figure 2: This figure is for the quenched case $\left(N_{f}=0\right)$. The top row shows the $\hat{\alpha}$-dependence of the average phase in the chiral limit for $v=0,1,2$. The solid lines are the RMT results for $N \rightarrow \infty$, and the data points are from RMT simulations at $N=20$. The bottom row shows the dependence on $\hat{m}$ for fixed $\hat{\alpha}=1$, with RMT simulations at $N=20$ and $N=80$. The deviations between the analytical results and the data points are simply finite- $N$ effects. The filled area represents the contribution of the $\Delta$-term specific to $v \neq 0$.
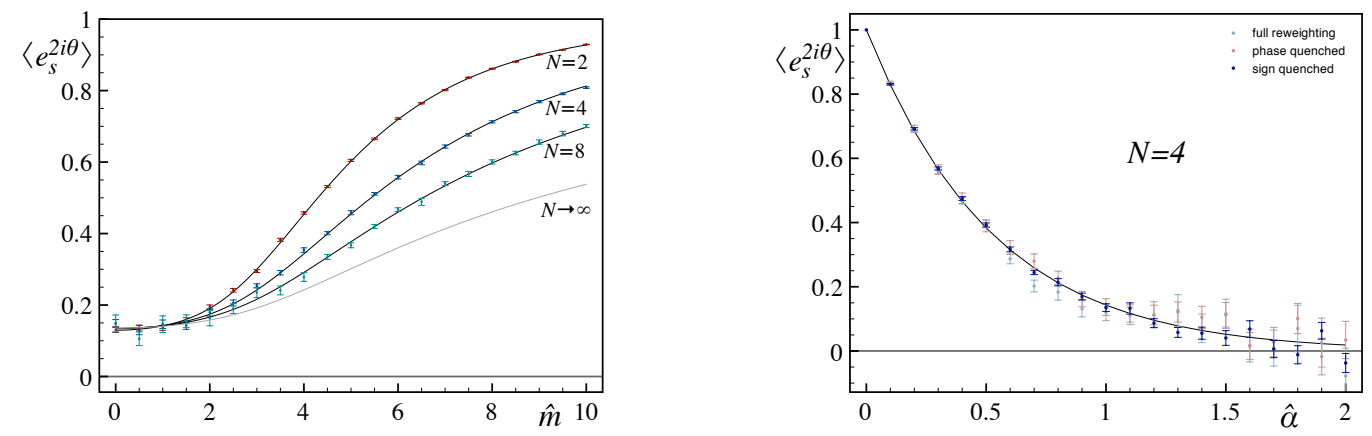

Figure 3: Dependence of the average phase on $\hat{m}$ at fixed $\hat{\alpha}=1$ (left) and on $\hat{\alpha}$ at fixed $\hat{m}=1$ (right), both for $N_{f}=2$ and $v=1$. The solid lines are the analytical results at finite $N$ [17], and the data points are from reweighted RMT simulations at the same value of $N$. In the left plot sign-quenched reweighting was used.

\section{Summary and outlook}

We have computed analytical results for the average phase of the fermion determinant from RMT and found that the sign problem becomes milder with increasing $|v|$. Our analytical results are valid for QCD in the microscopic regime. We have confirmed these results by extensive numerical simulations of RMT. Interesting projects for the future are the calculation of the distribution of the phase for arbitrary topology, lattice simulations at larger volume, and a study of the applicability of the sign-quenched algorithm (item 3 above) in other problems. 


\section{References}

[1] P. de Forcrand, Simulating QCD at finite density, PoS(LAT2009)010.

[2] K. Splittorff and J. J. M. Verbaarschot, Phase of the Fermion Determinant at Nonzero Chemical Potential, Phys. Rev. Lett. 98 (2007) 031601, [hep-lat / 0609076 ].

[3] K. Splittorff and J. J. M. Verbaarschot, The QCD sign problem for small chemical potential, Phys. Rev. D75 (2007) 116003, [hep-lat/ 0702011 ].

[4] JLQCD Collaboration, S. Aoki et. al., Two-flavor QCD simulation with exact chiral symmetry, Phys. Rev. D78 (2008) 014508, [arXiv: 0803 . 3197].

[5] J. C. R. Bloch and T. Wettig, Overlap Dirac operator at nonzero chemical potential and random matrix theory, Phys. Rev. Lett. 97 (2006) 012003, [hep-lat/ 0604020 ].

[6] J. C. R. Bloch and T. Wettig, Random matrix analysis of the QCD sign problem for general topology, JHEP 03 (2009) 100, [arXiv: 0812 . 032 4].

[7] J. Han and M. A. Stephanov, A Random Matrix Study of the QCD Sign Problem, Phys. Rev. D78 (2008) 054507, [arXiv:0805.1939].

[8] M. P. Lombardo, K. Splittorff, and J. J. M. Verbaarschot, Distributions of the Phase Angle of the Fermion Determinant in QCD, arXiv:0904.2122.

[9] M. P. Lombardo, K. Splittorff, and J. J. M. Verbaarschot, Fluctuations, correlations and the sign problem in QCD, PoS(LAT2009)171.

[10] S. Ejiri, Canonical partition function and finite density phase transition in lattice QCD, Phys. Rev. D78 (2008) 074507, [arXiv: 0804 .3227].

[11] J. Danzer, C. Gattringer, L. Liptak, and M. Marinkovic, A study of the sign problem for lattice QCD with chemical potential, arXiv:0907.3084.

[12] J. C. Osborn, Universal results from an alternate random matrix model for QCD with a baryon chemical potential, Phys. Rev. Lett. 93 (2004) 222001, [hep-th/ 0403131 ].

[13] G. Akemann and A. Pottier, Ratios of characteristic polynomials in complex matrix models, J. Phys. A37 (2004) L453-L460, [math-ph/ 0404068 ].

[14] M. C. Bergere, Biorthogonal Polynomials for Potentials of two Variables and External Sources at the Denominator, hep-th/0404126.

[15] G. Akemann, J. C. R. Bloch, L. Shifrin, and T. Wettig, Individual complex Dirac eigenvalue distributions from random matrix theory and lattice QCD at nonzero chemical potential, Phys. Rev. Lett. 100 (2008) 032002, [arXiv:0710.2865].

[16] JLQCD Collaboration, H. Fukaya et. al., Two-flavor lattice QCD simulation in the epsilon-regime with exact chiral symmetry, Phys. Rev. Lett. 98 (2007) 172001, [hep-lat/ 0702003 ].

[17] J. C. R. Bloch and T. Wettig, to be published. 